\title{
Hereditary disorders of connective tissue: A guide to the emerging differential diagnosis
}

\author{
Maureen Murphy-Ryan, BS $S^{I}$, Apostolos Psychogios, $M D^{2}$, and Noralane M. Lindor, $M D^{2}$
}

\begin{abstract}
Purpose: To create a practical desk reference for clinicians focused on the differential diagnosis of individuals presenting with features that suggest an inherited disorder of connective tissue. Methods: We searched the medical literature for distinct clinical entities that shared clinical features with Marfan syndrome and other classical inherited disorders of connective tissue. Results: Thirty-six distinct heritable disorders of connective tissue were identified that have overlapping features. These disorders were organized into two matrices according to clinical characteristics and according to causative genes. Conclusions: A broad differential diagnosis is emerging for individuals presenting with features suggestive of altered connective tissue. Recent advances in molecular genetics have aided in the delineation of these disorders. Genet Med 2010:12(6):344-354.
\end{abstract}

Key Words: connective tissue, aneurysm, dissection, aorta, vascular disease

$M$ any clinicians have a basic knowledge of Marfan syndrome, a relatively common genetic disorder of connective tissue with clinical manifestations involving the musculoskeletal, cardiovascular, respiratory, ophthalmologic, and cutaneous systems. ${ }^{1}$ However, it is less well known that there is an emerging and complex differential diagnosis to be considered for patients evaluated for a possible heritable disorder of connective tissue. Molecular genetic discoveries have greatly advanced the ability to correctly classify these disorders, and recent reviews have compared clinical features of subsets of the inherited connective tissue disorders. ${ }^{2-5}$ The aim of this article was to consolidate new clinical and genetic information in an accessible, comprehensive format to aide in the diagnostic evaluation of individuals who have features that may initially raise the possibility of a connective tissue disorder. The accompanying tables will provide the majority of necessary information to develop a thorough differential diagnosis. Limited text is included to add relevant syndrome-specific information that could not be adequately captured in table format.

\section{Background}

The constitution of the normal connective tissue varies from one tissue to another, both in the specific elements-collagen, elastin, fibrillin, fibulin, ${ }^{6}$ and others - and in the proportion of

From ${ }^{1}$ Mayo Medical School, Rochester, Minnesota; and ${ }^{2}$ Department of Medical Genetics, Mayo Clinic, Rochester, Minnesota.

Noralane M. Lindor, MD, E7B Mayo Clinic, Rochester, MN 55902; E-mail: nlindor@mayo.edu.

Disclosure: The authors declare no conflicts of interest.

Supplemental digital content is available for this article. Direct URL citations appear in the printed text and are provided in the HTML and PDF versions of this article on the journal's Web site (www.geneticsinmedicine.org).

Submitted for publication January 11, 2010

Accepted for publication March 27, 2010.

Published online ahead of print May 14, 2010.

DOI: 10.1097/GIM.0b013e3181e074f0 each element. Proteins that modify these component elementssuch as the metalloproteinases ${ }^{7}$ and lysyl hydroxylase 3 , which act on the structure of collagen fibers, and homocysteine, which can affect the integrity of fibrillin in elastic tissues-have also been implicated in disorders of connective tissue. In addition, mutations in genes that affect protein glycosylation are also implicated in the differential diagnosis of connective tissue disorders. Because each of these elements contribute to connective tissue structure in a number of different body locations, genetic defects in a single connective tissue component may manifest in more than one organ in the body (pleiotropy). This results in clinically overlapping multisystemic findings that may make diagnosis of a specific entity especially challenging.

Recent advances in molecular diagnostics have greatly increased our appreciation of the complexity of disorders of connective tissue. The fibrillinopathies illustrate this complexity. Marfan syndrome is but one clinical entity caused by FBNI mutations. Depending on the nature or location of the mutation within the FBN1 gene, different clinical entities may result including familial ectopia lentis; ascending aortic aneurysm; mitral valve, aorta, skeleton, and skin (MASS) syndrome; and Marfan syndrome. These allelic disorders have been grouped together as "fibrillinopathies" and are distinguished by their clinical presentations. ${ }^{8}$ As the prognosis and management is quite different between the various fibrillinopathies, precision in diagnosis is important. In light of the recent rapid accumulation of knowledge in the realm of genetics of connective tissue disorders, we sought to organize the clinical and molecular features in a way that help health care professionals who regularly encounter such disorders to establish a reasonable differential diagnosis to guide clinical evaluation and genetic testing.

\section{MATERIALS AND METHODS}

We developed a graphical format to consolidate the key clinical and genetic features of heritable disorders of connective tissue. Marfan syndrome was used as a platform for comparison. Each of the clinical features of Marfan syndrome, as specified by the revised Ghent Criteria, were subjected to PubMed and OMIM literature searches to identify other Mendelian syndromes or clinical entities that shared that feature. Thirty-six different clinical entities that might share one or more of the presenting features in Marfan syndrome were identified and included in the matrices, involving more than 40 different genes or gene loci. As some of these clinical entities are very new or very rare, only limited data may be available. Boxes are marked with an " $x$ " when that sign was fairly well established in the medical literature as a nonrandom association with that disorder. In Table 1, we present a list of clinical signs that were shared between at least two of the identified syndromes organized by organ system (available as Supplemental Digital Content 1, http://links.lww.com/GIM/A111). Table 2 shows the causative genes for these clinical entities (available as Supplemental Digital Content 2, http://links.lww.com/GIM/A112). This format best illustrates that there is no one-to-one correspondence between genes and disorders. 


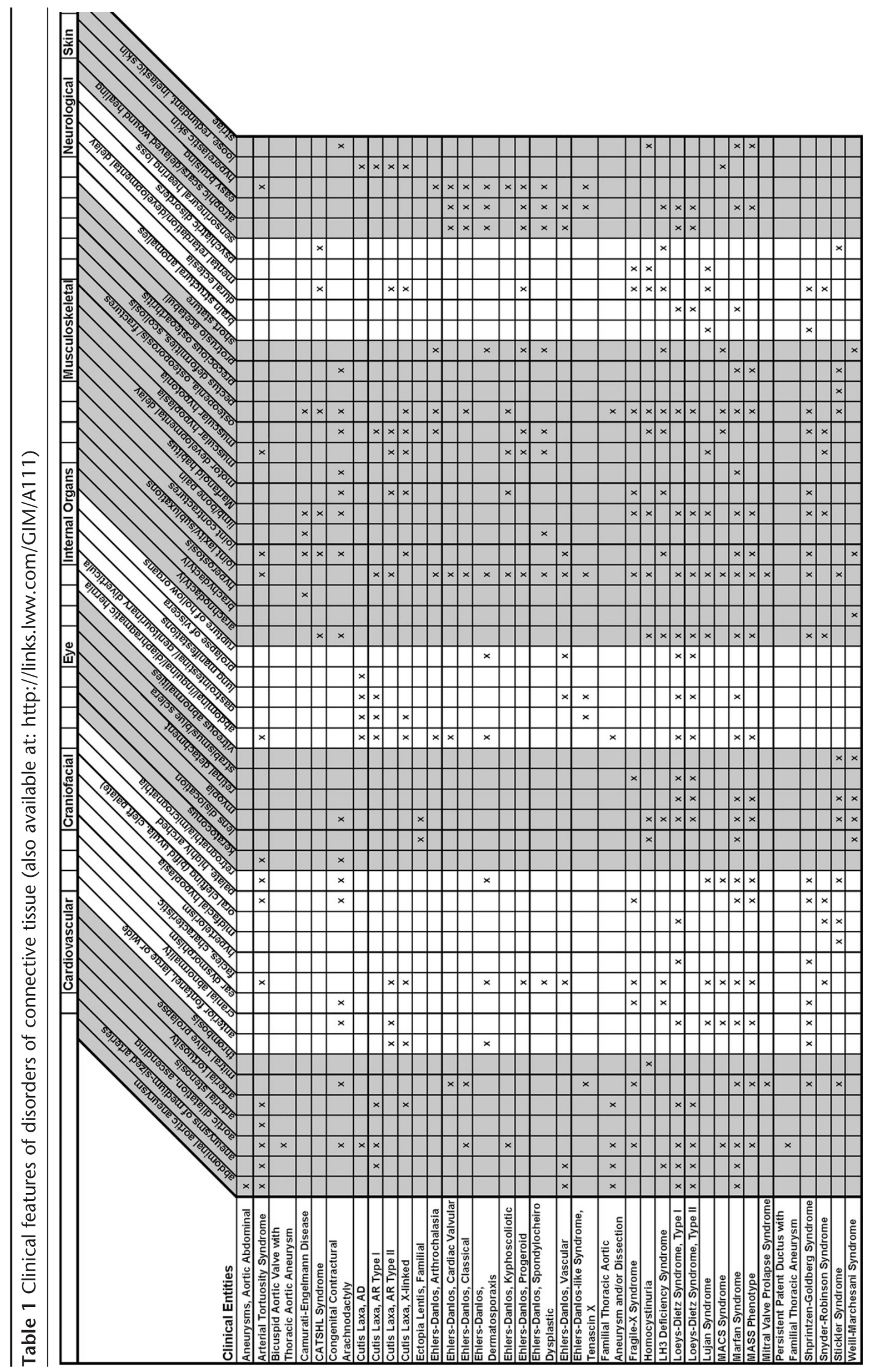




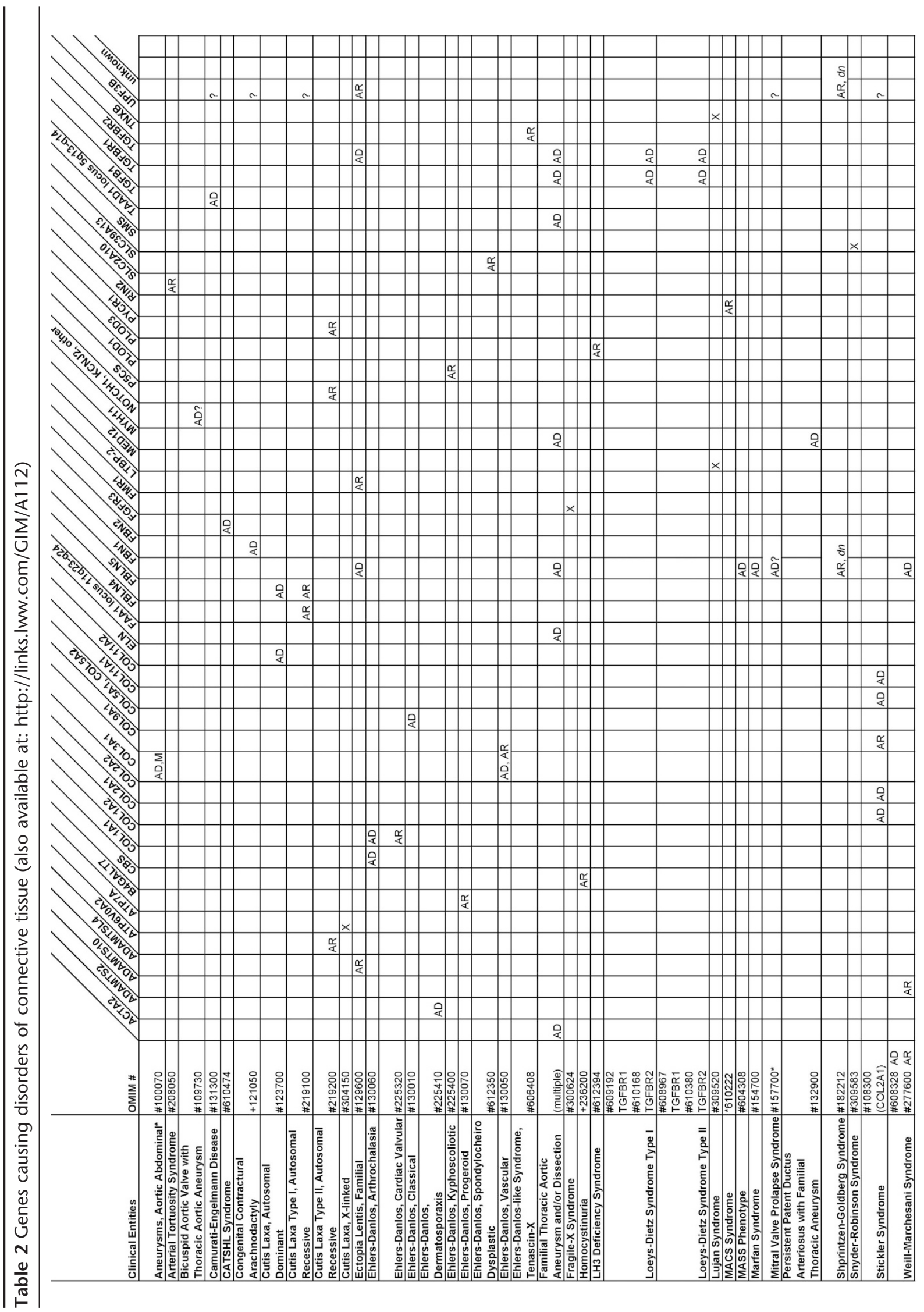




\section{RESULTS}

Listed alphabetically below are additional facts to supplement the main body of information in the tables.

\section{Aneurysms, aortic abdominal}

Aneurysms, aortic abdominal (AAA) may be present in isolation or as part of a syndrome such as vascular Ehlers-Danlos syndrome (EDS), Loeys-Dietz syndrome, or Marfan syndrome. Three loci for isolated AAA have been identified: 19q13, 4q31, and 9 p21. However, most AAAs are of multifactorial etiology. ${ }^{9}$

\section{Arterial tortuosity syndrome}

Arterial tortuosity syndrome is distinguished from forms of EDS by the generalized tortuosity of the arterial bed. Arterial tortuosity syndrome may include occasional manifestations such as inguinal and diaphragmatic hernias, intestinal elongation, keratoconus, macrocephaly, arachnodactyly, joint contractures, and hypotonia in addition to the more common features listed in Table 1 (Supplemental Digital Content 1, http://links.lww.com/GIM/A111). Characteristic facial features can include a distinctive elongated facies, micrognathia, small mouth, high arched palate, low set ears, long philtrum, beaked nose, down-slanting palpebral fissures, long ears, and sagging cheeks. ${ }^{10,11}$

\section{Bicuspid aortic valve with thoracic aortic aneurysm}

Bicuspid aortic valve (BAV) with thoracic aortic aneurysm (TAA) is often associated with aortic calcification, ${ }^{12}$ and research in mice and humans suggests that these conditions have a common etiology through genes such as NOTCH1. ${ }^{13-15}$ Individuals with normal tricuspid aortic valves in families with BAV/TAA can also be at risk for aortic valve calcification or TAA, especially if they carry a known familial mutation in NOTCH1. ${ }^{16}$ Clinical management suggestions for BAV/TAA may include pharmacologic therapy with beta-blockers and angiotensin converting enzyme inhibitors or angiotensin receptor blockers, aggressive hypertension control, annual imaging of ascending aortas with diameters $>4.0 \mathrm{~cm}$, and elective surgical repair of dilated aortas $>5.0 \mathrm{~cm}$ in diameter or $>4.5 \mathrm{~cm}$ with additional risk factors. ${ }^{16}$

\section{Camurati-Engelmann disease}

Camurati-Engelmann disease (CED) is a rare bone dysplasia, also known as progressive diaphyseal dysplasia, within the group of craniotubular hyperostosis disorders resulting from activating mutations in transforming growth factor $\beta 1$ (TGF- $\beta 1$ ), a bone growth promoter. Skeletal scintigraphy can be used detect CED even at early stages. In addition to pain in the extremities (the most common symptom), other diagnostic clinical features include a waddling gate, easy fatigability, and muscle weakness. Reduced subcutaneous fat and cranial nerve defects including hearing loss are reported in a subset of CED patients. ${ }^{17} \mathrm{CED}$ and Ribbing disease (OMIM no. 601477) are phenotypic variations of the same genetic disorder.

\section{CATSHL syndrome}

CATSHL syndrome stands for "camptodactyly, tall stature, and hearing loss." This rare syndrome was identified in a family with a missense mutation in the tyrosine kinase domain of FGFR3, predicted to result in a partial loss of function. ${ }^{18}$ Generally, strong genotype-phenotype correlations characterize FGFR-related disorders. Mutations in FGFR3 predominantly affect bones that develop by endochondral ossification, whereas mutations in closely related genes, FGFR1 and FGFR2, exert their primary effects on bones developing by intramembranous ossification. FGFR3 mutations may promote (as in the case of CATSHL) or inhibit (as in the achondroplasias) endochondral bone growth.

\section{Congenital contractural arachnodactyly}

Congenital contractural arachnodactyly (CCA) is also known as Beals-Hecht syndrome. The classic ear dysmorphism involves a "crumpled" appearance with a folded upper helix, although this feature is not always present. Medial degeneration of the proximal aorta leading to aortic aneurysm and possibly dissection is occasionally seen in CCA and should be closely monitored. Contractures of the knees and ankles may be present at birth and often improve with age. Flexion contractures in the small joints of the digits also occur, and hip contractures, thumb adduction, and clubfoot are less common. Paradoxical patellar laxity, keratoconus, and pyeloureteral junction stenosis have also been reported. A rare severe/lethal form of CCA has also been identified, which includes severe cardiovascular and gastrointestinal symptoms. ${ }^{19}$ Significant clinical differences have not been observed between FBN2-mutation-positive and FBN2negative patients, suggesting locus heterogeneity. ${ }^{20}$ Of FBN2positive patients, frequency of joint dislocation is significantly higher with FBN2 missense mutations compared to splice-site mutations. ${ }^{21}$ The proportion of de novo cases is unknown, but many patients diagnosed with CCA have an affected parent. ${ }^{22}$

\section{Cutis laxa, autosomal dominant}

Cutis laxa, autosomal dominant (ADCL) was originally thought to involve minor systemic manifestations; however, thoracic aortic aneurysmal disease, inguinal hernias, and emphysema are now recognized as serious potential clinical manifestations. ${ }^{23-25}$

\section{Cutis laxa, autosomal recessive type I}

Cutis laxa, autosomal recessive AR type I (ARCLI) has the poorest prognosis of the cutis laxas and is thought to be less common that ARCLII. The lung manifestations in ARCLI involve severe cardiopulmonary lesions, including infantile emphysema and/or supravalvular aortic stenosis. Clinical differences exist between ARCLI caused by FBLN4 and FBLN5; for example, only FBLN4 has been linked to collagen-related abnormalities such as bone fragility and aneurysms of mediumsized arteries. ${ }^{26,27}$ The genetic basis for the majority of ARCLI cases remains unknown. Recently, Morava et al. ${ }^{28}$ published a comprehensive comparison of the autosomal recessive cutis laxa syndromes.

\section{Cutis laxa autosomal recessive Type II}

Cutis laxa autosomal recessive Type II (ARCLII) often includes a characteristic facies with down-slanting palpebral fissures, a short nose, and a small mouth. Central nervous system and systemic involvement is variable, and microcephaly is often present. The skin phenotype tends to attenuate with age, whereas neurological symptoms may emerge or worsen later in life. The large anterior fontanel exhibits late closure. Many patients with ARCLII have a congenital defect of glycosylation (CDG type II, ATP6V0A2 mutation), whereas others have defects in proline biosynthesis associated with wrinkly skin, osteopenia, and progeroid appearance (PYCR1, P5CS mutations). ${ }^{29,30}$ Analysis of apolipoprotein C-III isoelectric focusing, demonstrating a disturbance of $O$-glycosylation, is diagnostic, and isoelectric focusing of transferrin may also be disturbed. Evaluation of protein glycosylation status is recommended for all children presenting with congenital wrinkled skin/cutis laxa, late fontanel closure, developmental delay, and variable CNS 
involvement. Wrinkly skin syndrome (OMIM no. 278250) and ARCLII are part of a spectrum of phenotypic variation caused by defects in a single gene. ${ }^{31}$ The phenotype may also overlap with gerodermia osteodysplasia (OMIM no. 231070) and de Barsy syndrome (OMIM no. 219150).

\section{Cutis laxa, X-linked}

Cutis laxa, X-linked (XLCL) is also known as occipital horn syndrome, due to the presence of diagnostic bilateral, symmetric wedge-shaped bony outgrowths beside the foramen magnum at the site of trapezius and sternocleidomastoid muscle attachment. There may be hyperextensibility of wrist and interphalangeal joints but contractures of elbow and knee. Facial features may include a hooked nose and long philtrum, which are characteristic of cutis laxa in general. Failure to thrive may be due to chronic diarrhea, malabsorption, congenital hydronephrosis, or urethral and bladder diverticulae. ${ }^{27}$ Mental retardation may or may not be present. XLCL is a copper transport disease allelic with Menkes Disease (OMIM no. 309400), often sharing the feature of brittle hair. Serum ceruloplasmin may be low or normal, and lysyl oxidase activity may also be decreased.

\section{Ectopia lentis, familial}

Ectopia lentis, familial is caused by mutations in FBN1 that are predicted to disrupt normal disulfide bond formation and native protein structure. ${ }^{30}$ Glaucoma can be present secondary to lens subluxation. Although mutations in FBN1 result in autosomal dominant isolated ectopia lentis, autosomal recessive variations have also been described, some of which are due to mutations in $L T B P-2^{32}$ and ADAMTSL4 ${ }^{33}$. Three families with autosomal recessive ectopia lentis due to mutations in $L T B P-2$ exhibited mild-to-moderate osteopenia in carriers and affected individuals as well as a high arched palate, although no other features overlapping with Marfan syndrome were present, whereas all cases with $L T B P$-2-null mutations in a fourth family had Marfanoid skeletal and joint features without osteopenia. ${ }^{32}$ Ectopia lentis may also occur in combination with mild skeletal symptoms, mitral valve prolapse, or nonprogressive aortic root dilatation, especially when due to mutations in FBN1. Some patients diagnosed with familial ectopia lentis may develop aortic aneurysm later in life and could be considered to have late forms of Marfan syndrome. Ectopia lentis has also been identified in a subset of patients with TGFBR2 mutations and minimal skeletal findings. ${ }^{34}$

\section{Ehlers-Danlos syndrome, arthrochalasia type (EDS type VIIA, VIIB)}

Ehlers-Danlos syndrome, arthrochalasia type (EDS type VIIA, VIIB) is distinguished from other types of EDS by a high frequency of early-onset congenital hip dislocation, extreme joint laxity with recurrent joint subluxations (large and small, particularly of the hips), and minimal skin involvement. Hyperelasticity of skin is usually mild when present. Individuals with arthrochalasia type of EDS may exhibit a history of frequent fractures, a phenotypic overlap with osteogenesis imperfecta, and short stature may also be associated. ${ }^{35}$ The pathologic basis of this disorder is an inability of $\alpha-1$ or $\alpha-2$ chains of type I procollagen to be converted to collagen due to mutations altering the procollagen cleavage site.

\section{Ehlers-Danlos syndrome, cardiac valvular type}

Ehlers-Danlos syndrome, cardiac valvular type can have the typical skin and joint involvement of classical EDS (although not always) as well as an increased risk for cardiac valvular involvement. This is a very rare variant of EDS, with only a handful of patients having been described to date. The molecular basis is a complete absence of pro $\alpha 2(\mathrm{I})$ collagen chains, resulting from homozygosity or heterozygosity for COL1A2 null mutations. ${ }^{36}$ Cardiac-valvular EDS is a relatively mild variant on the spectrum of clinical phenotypes resulting from COL1A2 mutations, which also includes osteogenesis imperfecta. Unlike for osteogenesis imperfecta, no skeletal abnormalities are observed in cardiac valvular EDS. The cardiac valvular type of EDS may overlap with mild form of hypermobility type EDS during childhood, with cardiac valvular complications emerging during adulthood. ${ }^{37}$

\section{Ehlers-Danlos syndrome, classical type (EDS type I, II)}

Ehlers-Danlos syndrome, classical type (EDS type I, II) features skin that is often characterized as smooth and velvety. Atrophic scarring is a key dermatological finding. Typical facial features may include epicanthic folds, excess eyelid skin, and a prematurely aged appearance. It is distinct from the hypermobility type, which has much more subtle skin manifestations. Bruising may be the presenting symptom in pediatric patients. ${ }^{38}$ Type V collagen defects (COL5A1 and COL5A2) are identified in $\sim 50 \%$ of classical EDS cases. For the major and minor diagnostic features of the six commonly recognized types of EDS, see the revised nosology created by the Ehlers-Danlos National Foundation (USA) and Ehlers-Danlos Support Group (UK). ${ }^{39}$

\section{Ehlers-Danlos syndrome, dermatosparaxis type (EDS type VIIC)}

Ehlers-Danlos syndrome, dermatosparaxis type (EDS type VIIC) is characterized by extreme skin fragility. Patients with dermatosparaxis type EDS may exhibit a delayed onset of a typical phenotype, and at-risk infants may thus require follow-up during the first 2 years. Facial dysmorphism may include eyelid fullness, epicanthal folds, and down-slanting palpebral fissures. Blue sclera may be observed. The deciduous and permanent dentition may exhibit a variety of abnormalities. ${ }^{40}$ Similar to the arthrochalasia type of EDS, the dermatosparaxis type is caused by deficiencies in the processing of procollagen into collagen. Although the arthrochalasia type of EDS is caused by mutations in COL1A1 or COL1A2, the dermatosporaxis type involves mutations in the gene for the protease ADAMTS-2. ${ }^{41}$

\section{Ehlers-Danlos syndrome, kyphoscoliotic type (EDS type VI)}

Ehlers-Danlos syndrome, kyphoscoliotic type (EDS type VI) is distinguished from other types of EDS by gross motor developmental delay resulting from muscular hypotonia combined with joint laxity. Because of these clinical features, the differential diagnosis of infants with kyphoscoliotic EDS may initially include congenital muscular dystrophies, congenital myopathies, and lower motor neuron diseases. Adolescents with this form of EDS may present with generalized muscle weakness in addition to the reduced muscle tone in rest of hypotonia. ${ }^{42}$ Kyphoscoliotic EDS should be considered if results of neuromuscular tests come back normal, contractures are absent, and Marfanoid habitus is present. Diagnosis of kyphoscoliotic EDS can be biochemically confirmed by a significantly increased ratio of total urinary lysyl pyridinoline to hydroxylysyl pyridinoline. 43,44 


\section{Ehlers-Danlos syndrome, progeroid type}

Ehlers-Danlos syndrome, progeroid type is a congenital disorder of glycosylation, such as type II AR cutis laxa. ${ }^{45}$ As its name suggests, this type of EDS may be characterized by a distinctive progeroid facies as well as facial skin wrinkling, fine curly hair, scanty eyebrows and eyelashes, and downslanting palpebral fissures. Developmental delay and skeletal abnormalities are also typical. Skin may be loose but still elastic. A spectrum of severity is seen in the skin phenotype and may be as mild as slight facial wrinkling and no remarkable skin laxity. ${ }^{46}$

\section{Ehlers-Danlos syndrome, spondylocheirodysplastic type}

Ehlers-Danlos syndrome, spondylocheirodysplastic type (SCD-EDS) features skin that is described as smooth, velvety, thin, fragile, and translucent with atrophic (cigarette-paper) scarring, as in classical EDS. Over the hands and feet, the skin can appear wrinkled and prematurely aged, and the fingers are described as slender and tapering. Small joint contractures and substantial atrophy of the thenar and hypothenar muscles may limit fine motor skills and gives the hands a similar appearance to that seen in LH3 deficiency syndrome. Growth retardation occurs postnatally. Protruding eyes with down-slanting palpebral fissures and bluish sclera are characteristic of this disorder. Flat feet (pes planus), a common feature of connective tissue disorders, is also characteristic of SCD-EDS. Persons with SCD-EDS may exhibit a distinctive waddling gait and experience pain in the knees and hips when walking. ${ }^{47}$

\section{Ehlers-Danlos syndrome, vascular type (EDS type IV)}

Ehlers-Danlos syndrome, vascular type (EDS type IV) stems from a reduction or absence of normal type III collagen within the tunica media of large elastic arteries. This predisposes persons to abnormalities in major blood vessels and resultant aneurysms, spontaneous vascular rupture, dissections, and arteriovenous fistulas. Individuals with vascular EDS exhibit extreme bruisability. Commonly ruptured hollow organs include the intestines, particularly the colon, and the uterus, making pregnancy highly risky for women with vascular EDS. Lung manifestations, although less common, have recently been reported and may include recurrent hemoptysis, spontaneous pneumothorax, or bulla and bleb formation. Distinctive facial features inconsistently may include acrogeria, characterized as a slender facies with prominent bones; sunken cheeks; thin, pinched nose; bulging eyes; and thin lips. ${ }^{48,49}$ Skin hyperelasticity and joint hypermobility are limited in vascular EDS. The majority of familial vascular EDS cases are inherited in an autosomal dominant fashion, although a case of recessively inherited vascular EDS has been identified. ${ }^{50}$ There is significant phenotypic overlap between vascular EDS and familial arterial aneurysms. It is important to distinguish between Loeys-Dietz Syndrome and vascular EDS because of the significantly higher rate of intraoperative mortality associated with the latter. ${ }^{51}$

\section{Ehlers-Danlos-like syndrome, tenascin $\mathrm{X}$ deficiency}

Ehlers-Danlos-like syndrome, tenascin X (TNX) deficiency is characterized by hypermobile joints, hyperelastic skin, and very easy bruising but without the atrophic scarring or poor wound healing seen in classical EDS. Other significant medical problems occurring in persons with this disorder include severe diverticular intestinal disease (e.g., pancolonic) with ruptured diverticula, gastrointestinal bleeding, rectal prolapse, mitral valve prolapse requiring valve replacement, and chronic obstructive airway disease. Abnormal nerve conduction studies indicating polyneuropathy are common and significantly more frequent in tenascin-X deficient EDS compared with other forms of EDS. ${ }^{52}$ Although this is a recessive condition, up to $2 / 3$ of females heterozygous for TNX deficiency may have isolated joint hypermobility. Less invasive alternatives to endoscopy should be considered for patients with TNX deficient EDS because of the theoretical risk of bowel perforation. Baseline pulmonary testing is recommended for all patients with TNX deficient EDS, and smoking patients should be strongly encouraged to stop because of the risk for COPD. ${ }^{53}$ Contiguous gene deletions involving both tenascin $\mathrm{X}$ and 21-hydroxylase genes can result in congenital adrenal hyperplasia with tenascin $\mathrm{X}$ deficiency. ${ }^{54,55}$

\section{Familial thoracic aneurysm and/or dissection}

Familial thoracic aneurysm and/or dissection (FTAAD) results in heritable aortic dilatation, aneurysm, and dissection. ${ }^{56}$ Cerebral vessels may also be affected with fusiform aneurysms or tortuosity. Multiple genes, the majority of which act in an autosomal dominant manner, cause FTAAD, and linkage to other loci indicates that additional genes await discovery. Smooth muscle cell contraction has been found to be important in maintaining the structural integrity of the thoracic/ascending aorta, and mutations in the genes coding for smooth muscle cell $\alpha$-actin (ACTA2) and $\beta$-myosin heavy chain (MYH11) have been identified as causes of FTAAD. ${ }^{57}$ Mutations in $A C T A 2$ are the most common cause of FTAAD and may be associated with livedo reticularis, iris flocculi, PDA, and BAV. FTAAD caused by TGBFR mutations often involves skeletal manifestations of a connective tissue disorder (such as joint hypermobility, pes planus, dolichocephaly, or a highly arched palate) that do not meet diagnostic criteria for a particular syndrome. Differences in clinical presentation between individuals with FTAAD due to TGFBR1 and TGFBR2 have been identified, including an increase in cancer incidence with TGFBR1 compared with TGFBR2 and better survival for women with vascular disease due to TGFBR1 mutation with no gender difference seen in TGFBR2.58 An inclusive overview of the molecular basis of FTAAD was published by Milewicz et al. ${ }^{59}$ in 2008.

\section{Fragile- $X$ syndrome}

Fragile-X syndrome, also known as Martin-Bell syndrome, is the most common cause of X-linked mental retardation. The syndrome includes a variety of clinical features of disordered connective tissue, and skin and cardiac tissues from individuals with Fragile-X syndrome demonstrate elastin fibers that are aberrantly distributed and structured. ${ }^{60,61}$ Pes planus is a common musculoskeletal finding in addition to those listed in Table 1 (Supplemental Digital Content 1, http://links.lww.com/GIM/A111). Facial features may be subtle; the characteristic facies is long with a high forehead, large jaw and prominent chin, and ears are characteristically large and low set. Strabismus (but not blue sclera) was present in roughly half of affected individuals in one study. ${ }^{60}$ Affected individuals may display features of autism. Behavioral-cognitive manifestations most commonly include a combination of poor eye contact, perseverative speech, echolalia, poor attention span, hyperactivity, and unusual hand mannerisms; a patient who presents with this characteristic facies, joint laxity, macroorchidism, a family history of mental retardation, and any of the psychiatric manifestations listed above should be evaluated for Fragile-X syndrome. Female Fragile X premutation carriers are at risk for premature ovarian failure. 
Premutations are also associated with a syndrome of tremor and ataxia. $^{62}$

\section{Homocystinuria}

Homocystinuria is due to cystathionine beta-synthase deficiency (CBS). There is a high risk for morbidity and mortality due to vascular thrombosis and stroke. ${ }^{63}$ Clinical clues to the diagnosis include generalized hypopigmentation, pancreatitis, malar flush, and livedo reticularis. Subluxation of the lens is usually downward in homocystinuria, whereas in Marfan syndrome, the lens is generally dislocated upward. Neuropsychiatric disease is common in untreated patients.

\section{LH3 deficiency syndrome}

LH3 deficiency syndrome can manifest with a characteristic facies of shallow orbits, a small nose, down-turned mouth corners, a flat facial profile, and low-set, simplified ears. Osteopenia and fractures may be present, and growth retardation is both prenatal and postnatal. In addition to the features listed in Table 2 (Supplemental Digital Content 2, http://links.lww.com/GIM/A112), nonscarring skin blistering, hypoplastic nails, spontaneous hemorrhage, and cataracts were present. Progressive scoliosis was identified but not pectus deformities. Clubfoot (talipes equinovarus), small joint contractures, and atrophy of the thenar and hypothenar muscles are similar to the spondylocheirodysplastic form of EDS. The clinical features in LH3 (lysyl hydroxylase 3) deficiency are thought to stem from the loss of the glucosyltransferase activity of LH3, which normally modifies the pyridinoline crosslinks of collagen extracellularly via hydroxylysine glycosylation. Thus, the absence of the disaccharide derivative of pyridinoline (Glc-Gal-PYD) in urine is biochemically diagnostic but not yet clinically available. ${ }^{64}$

\section{Loeys-Dietz syndrome}

Loeys-Dietz syndrome has a continuum of presentations that generally include both vascular and skeletal findings and is divided into two types based on the third major system involved. Craniofacial findings are seen particularly in LDS type I, which accounts for about three quarters of LDS. Cutaneous findings are seen in the quarter of LDS patients diagnosed with LDS II. These features of organ rupture and vascular events are similar to those seen in vascular EDS. As in classical EDS, the skin may be described as translucent and velvety in LDS II. Genotype-phenotype correlations do not explain the distinction between LDS I and LDS II. TGFBRI mutations account for $25 \%$ of LDS patients and TGFBR2 mutations account for $75 \%$ of LDS patients with no correlation to type. ${ }^{65}$ In both types of LDS, the vascular manifestations tend to be more severe than in Marfan syndrome and require more aggressive screening and treatment. Pneumothorax is the primary lung manifestation of Loeys-Dietz syndrome. Organs including the spleen, bowel, and uterus are at risk for rupture. Hernias are often recurrent. In addition to dural ectasia, neuroradiologic findings may include Arnold-Chiari type I manifestation, but this may be relatively rare. A minority of LDS patients will have developmental delay.

\section{Lujan syndrome}

Lujan syndrome is also known as Lujan-Fryns syndrome. The psychiatric manifestations may include a combination of hyperactivity, emotional lability, shyness, aggressiveness, autistic mannerisms, and psychoses. ${ }^{66}$ The major structural defect of the brain is some degree of corpus callosum agenesis. The characteristic facies of Lujan syndrome is long and thin with a tall forehead, short philtrum and sometimes micrognathia. Other distinctive features not mentioned in Table 2 include a hyper- nasal voice and macrocephaly. For more information on the differential diagnosis for Lujan Syndrome, see Van Buggenhout and Fryns ${ }^{67}$ and Staholpu et al. ${ }^{68}$ FG syndrome type 1, also known as Opitz-Kaveggia syndrome type 1, is allelic to Lujan syndrome ${ }^{69}$ and shares many clinical features, but none that are characteristically marfanoid. Anal anomalies or constipation are restricted to FG Syndrome, and tall stature, long hands and fingers, and hypernasal speech resulting from a high nasal root are more characteristic of LS. ${ }^{70}$ Lujan syndrome and FG syndrome are caused by mutations in $M E D 12$. Mutations in $U P F 3 B$ have also been identified in families with Lujan-Fryns phenotype and the FG phenotype. ${ }^{71}$

\section{MACS syndrome (macrocephaly, alopecia, cutis laxa, and scoliosis)}

MACS syndrome (macrocephaly, alopecia, cutis laxa, and scoliosis) is a newly described hereditary disorder of connective tissue based on three members of two related families. ${ }^{72}$ The clinical presentation is expected to evolve as more individuals with RIN2 mutations are identified. Macrocephaly and retrognathia are the predominant cranial abnormalities. Alopecia refers to the sparse scalp hair seen in this disorder. Cutis laxa is most notable in the face and mild ichthyosis may also be present. Severe scoliosis appears in the second decade of life (not at birth as in kyphoscoliotic EDS), resulting in short stature. The characteristic facies includes downslanting palpebral fissures, puffy eyelids, sagging cheeks, and everted lower lip. Ortho- and periodontal manifestations include abnormal positioning of the teeth (irregularly placed or unerupted) and gingival hyperplasia. The cutaneous phenotype becomes more pronounced with age in contrast to ARCLII, in which skin manifestations attenuate over time. Skin biopsy of affected individuals revealed fibulin-5 deficiency and a decrease in dermal microfibrils. Facial features resemble GAPO (growth retardation, pseudoanodontia, optic atrophy) syndrome (OMIM no. 230740), a rare autosomal recessive disorder that is phenotypically more severe and includes eye abnormalities, and the dermatosparaxis type of EDS, which also shares gingival hyperplasia but differs in having transparent, easy bruising skin.

\section{Marfan syndrome}

The diagnosis of Marfan syndrome is a clinical diagnosis, relying on the revised Ghent consensus criteria. The diagnosis requires major clinical signs in at least two systems and milder or less specific clinical signs in a third system. ${ }^{73}$ Skeletal findings include tall stature relative to other family members (attributed at least in part to disproportionately long limbs), long digits (arachnodactyly), anterior chest deformity including protrusion (pectus carinatum) or sunken appearance (pectus excavatum) of the sternum and anterior ribs which is related to overgrowth of ribs, joint laxity or contractures, scoliosis, and craniofacial manifestations including highly arched palate, crowded teeth, and overbite. Dural ectasia is a major diagnostic clinical criterion involving the lumbosacral spine. Ophthalmologic findings include myopia, lens subluxation (ectopia lentis), and increased axial globe length and corneal flatness. Cardiovascular findings include mitral valve prolapse, mitral regurgitation, aortic regurgitation, and dilatation of the aortic root at the level of sinuses of Valsalva that may result in an aneurysm and/or dissection. Cutaneous features include striae and recurrent hernia formation. Respiratory involvement may manifest as pulmonary blebs, which predispose to pneumothorax. FBN1 mutations are inherited in about two-thirds of cases and rise de novo in the remainder. Hundreds of FBN1 mutations have been 
described, with the number continuing to grow. Few genotypephenotype correlations have been established because of the extreme allelic heterogeneity within Marfan syndrome and genetically related disorders. ${ }^{74-77}$ Although there is no known genetic heterogeneity in Marfan Syndrome, TGFBR2 mutations have also been recognized in patients with Marfan-like phenotypes. Clinical outcomes appear similar to those of patients with FBN1 mutations, with prognosis in either group depending more on the clinical disease expression and treatment than the responsible gene. Patients with FBN1 mutations were found to have more extensive skeletal involvement, whereas patients with TGFBR2 mutations had more severe aortic phenotypes overall. ${ }^{34}$

\section{MASS phenotype}

MASS phenotype indicates the involvement of the MASS. The aortic enlargement is borderline and nonprogressive, and the skin and skeletal findings are nonspecific. These clinical characteristics overlap significantly with those of Marfan syndrome and provide clear evidence of a systemic extracellular matrix defect but are not sufficient for a diagnosis of Marfan syndrome. Thus, the MASS phenotype is part of the clinical continuum of FBN1 disorders that ranges from Marfan syndrome to isolated features such as ectopia lentis. ${ }^{78}$ Consequently, it is difficult to distinguish MASS phenotype from the early stages of Marfan syndrome when assessing individuals, particularly children, in the absence of family history. Intermittent cardiovascular monitoring is therefore indicated.

\section{Mitral valve prolapse syndrome}

Mitral valve prolapse (MVP) syndrome refers to cases of idiopathic MVP in which other cardiac and connective tissue disorders have been ruled out. MVP syndrome is associated with systemic features that may include chest pain, dyspnea, thoracic cage deformity (including narrow A-P diameter), dysrhythmia, mild joint laxity, and long limbs. Prolapse occurs when the leaflets of the mitral valve billow into the left atrium and is estimated to be present in $2.4 \%$ of the general population, exhibiting age- and sex-dependent penetrance. ${ }^{79}$ MVP may be sporadic or familial, isolated, or as part of a syndrome. ${ }^{80}$ To date, no specific genes for MVP have been described, although three autosomal and one X-linked loci have been identified. Prognosis in MVP syndrome is better than for MVP in Marfan syndrome, with significantly lower risk for mitral regurgitation.

\section{Persistent patent ductus arteriosis with familial thoracic aneurysm}

Persistent patent ductus arteriosis with familial thoracic aneurysm (PDA with FTA) is a monogenic pathophysiologic entity within the heterogenous group of TAA disease. It is caused by mutations in a specific region of the smooth muscle cell myosin heavy chain gene MYH11, which are associated with marked aortic stiffness even in nonsymptomatic individuals with the disease haplotype. ${ }^{81-83}$ Risk in patients with PDA with FTA may be assessed through noninvasive measurement of aortic compliance and distensibility using MRI. FTA without PDA is also caused by MYH11 mutations. MYH11 is only rarely involved in sporadic isolated PDA. ${ }^{84}$

\section{Shprintzen-Goldberg syndrome}

Shprintzen-Goldberg syndrome (SGS) shares many clinical manifestations with MFS and LDS, suggesting that SGS may also act through disturbance of TGF- $\beta$ signaling pathways, although no unique genetic loci have yet been established. The identification of TGFBR1, TGFBR2, and FBN1 mutations in some patients diagnosed with SGS suggests that recognition of SGS as a separate pathogenetic entity may be inappropriate, although the issue continues to be controversial. ${ }^{85-87}$ The characteristic facies in SGS can include hypertelorism, down-slanting palpebral fissures, a highly arched palate, micrognathia, proptosis (protruding eyes), and low set, posteriorly rotated ears. ${ }^{88}$ The presence of this characteristic facies, craniosynostosis, and mental retardation are the major features used to distinguish SGS clinically. Other features include Chiari malformation, rib anomalies, and equinovarus deformity (clubfeet). SGS is distinct from Goldberg-Shprintzen syndrome (OMIM no. 609640) and Shprintzen syndrome (OMIM no. 192430).

\section{Snyder-Robinson syndrome}

Snyder-Robinson syndrome is a rare X-linked mental retardation syndrome with marfanoid skeletal features with similarities to Lujan syndrome. Other characteristic features of this syndrome include facial asymmetry with a prominent lower lip, nasal voice, unsteady gait, nonspecific movement disorder, EEG abnormalities, seizures, and diminished muscle bulk. ${ }^{89-91}$

\section{Stickler syndrome}

Stickler syndrome is a relatively common connective tissue disorder. Cataracts and glaucoma may accompany the other ocular findings in Table 2 (Supplemental Digital Content 2, http://links.lww.com/GIM/A112). Mild spondyloepiphyseal dysplasia may also be present, and the hearing loss may be conductive and sensorineural. A flat facies and enlarged knees are also frequently seen. The presence of some clinical features_-including retinal detachment, cataract, sensorineural hearing loss, early-onset degenerative joint disease, and certain skeletal abnormalities - is a function of age. Molecular analysis may assist in the diagnosis of the disorder but is not very useful in predicting the phenotypic expression of the disease, except in the case of ocular manifestations. ${ }^{92}$ Two vitreoretinal phenotypes are clinically recognized. ${ }^{93}$ Mutations in COL2A1 are associated with a type 1 "membranous" vitreous phenotype. Because exon 2 of COL $2 A 1$ is preferentially expressed in the eye, mutations in this exon result in an isolated type 1 vitreous defect. ${ }^{94}$ The less common type II "beaded" vitreous phenotype is associated with mutations in COL11A1. Mutations in COL11A2 produce a Stickler phenotype lacking vitreous manifestations, because COL11A2 is not expressed in this tissue. ${ }^{95}$

\section{Weill-Marchesani syndrome}

Weill-Marchesani syndrome (WMS) exhibits significant clinical homogeneity despite its genetic heterogeneity. In comparing autosomal dominant $(\mathrm{AD}$ - due to $F B N 1)$ and autosomal recessive (AR - due to ADAMTS10) WMS, no significant difference was found for the incidence of most clinical features. ${ }^{96}$ There was approximately a $20 \%$ difference in incidence between AR and AD WMS for microspherophakia and cardiac anomalies (more prevalent in AR WMS) as well as joint limitations and ectopia lentis (more prevalent in AD WMS), ${ }^{97}$ although these characteristics are commonly seen in both forms of WMS. Further complicating differentiation between AR and AD WMS based on family history, some heterozygotes for AR WMS display mild clinical manifestations of the disease, including short stature, brachydactyly, and abnormal gonioscopic findings. In addition to the eye manifestations listed in Table 2 (Supplemental Digital Content 2, http://links.lww.com/GIM/A112), shallow orbits and glaucoma can be features of WMS. Congenital cardiac abnormalities reported for patients with WMS include MVP, prolonged QT interval, and pulmonary and aortic valve 
stenoses. Patients with WMS and a history of palpitation, lightheadedness, dizziness or syncope should be evaluated for prolonged QTc, as it may be associated with serious arrhythmia. ${ }^{98}$ The differential diagnosis of WMS includes Hunter-MacDonald Syndrome. ${ }^{99}$

\section{DISCUSSION}

The cornerstones for establishing a diagnosis are a personal medical history, a family history extended through third-degree relatives, and a comprehensive genetics physical examination, with special attention to potential dysmorphic features, cardiac murmurs, body proportions, joint mobility, and skin characteristics. This article is meant to help guide the development of an informed differential diagnosis and suggest possible targets for further clinical evaluation and genetic testing. Considerations for specific testing in heritable disorders of connective tissue may include the following:

- Evaluation by an ophthalmologist with knowledge of disorders of connective tissue to assess abnormalities of the lens, retina, vitreous, and refraction.

- Radiographic imaging, including transthoracic or esophageal echocardiography, with careful, serial measurements of the aortic root at the level of the sinuses of Valsalva to compare with normal references for age and/or body mass index and evaluation of the aortic arch.

- CT or MR angiogram of the cerebral, neck, thoracic, abdominal, and pelvic arteries. An abnormality such as dissection, dilation, tortuosity, or aneurysm in any one artery should prompt a systematic assessment of all major arteries.

- A skeletal survey that may help evaluate underlying skeletal dysplasia, acetabular abnormalities, hyperostosis, or osteopenia.

- A bone densitometry study may be useful for identifying osteopenia.

- Special spine films, to help accurately diagnose the degree of any scoliosis.

- Brain imaging that may be indicated in individuals with neurologic signs or symptoms but not usually in individuals lacking such neurological features.

- Audiologic assessment, recommended when disorders with hearing loss are included in the differential diagnosis list.

- Clinical molecular testing when available for genes included in Table 2 (Supplemental Digital Content 2, http://links.lww.com/GIM/A112) associated with the disorders under consideration in the differential diagnosis. Current information on test availability and links to the laboratories offering testing can be found at Genetests.org.

Genetic disorders of connective tissue comprise a rapidly expanding list of Mendelian syndromes that can manifest with significantly overlapping clinical findings, especially involving the musculoskeletal, cardiovascular, respiratory, ophthalmologic, and cutaneous systems. Diagnostic accuracy is most critical in serious but highly treatable disorders. Such disorders include homocystinuria and potentially, with the recent development of beta-blocker therapy or angiotensin II receptor antagonist like losartan as potential therapies to limit the risk of further vessel dilatation or dissection in Marfan syndrome and Loeys-Dietz syndrome. ${ }^{100,101}$ An accurate diagnosis can guide the physician and patient in monitoring for the progression of known symptoms or emergence of new symptoms, identifying high-risk situations, and identifying other at-risk family members. Standard management recommendations have been published for the more common inherited connective tissue disorders such as Marfan syndrome, and the importance of diagnostic accuracy will increase as further disorder-specific evidencebased treatment regimes are developed. Diagnostic accuracy may improve the quality of life for patients, even those with disorders for which no major treatment has yet been developed. The matrices constructed from this project may be of clinical use to geneticists as well as other clinicians who encounter patients with apparent alterations affecting connective tissue.

\section{REFERENCES}

1. Pyeritz R, McKusick VA. The Marfan syndrome: diagnosis and management. N Engl J Med 1979;300:772-777.

2. Summers KM, West JA, Peterson MM, Stark D, McGill JJ, West MJ. Challenges in the diagnosis of Marfan syndrome. Med J Aust 2006;184:627-631.

3. Pearson GD, Devereux R, Loeys B, et al. Report of the National Heart, Lung, and Blood Institute and National Marfan Foundation Working Group on research in Marfan syndrome and related disorders. Circulation 2008; 118:785-791

4. Callewaert B, Malfait F, Loeys B, De Paepe A. Ehlers-Danlos syndromes and Marfan syndrome. Best Pract Res Clin Rheumatol 2008;22:165-189.

5. Mizuguchi T, Matsumoto N. Recent progress in genetics of Marfan syndrome and Marfan-associated disorders. J Hum Genet 2007;52:1-12.

6. Argraves WS, Greene LM, Cooley MA, Gallagher WM. Fibulins: physiological and disease perspectives. EMBO Rep 2003;4:1127-1131.

7. Porter S, Clark IM, Kevorkian L, Edwards DR. The ADAMTS metalloproteinases. Biochem $J$ 2005;386(Pt 1):15-27.

8. Whiteman P, Hutchinson S, Handford PA. Fibrillin-1 misfolding and disease. Antioxid Redox Signal 2006;8:338-346.

9. Kuivaniemi H, Shibamura H, Arthur C, et al. Familial abdominal aortic aneurysms: collection of 233 multiplex families. J Vasc Surg 2003;37:340 345 .

10. Faiyaz-Ul-Haque M, Zaidi SH, Wahab AA, et al. Identification of a p.Ser81Arg encoding mutation in SLC2A10 gene of arterial tortuosity syndrome patients from 10 Qatari families. Clin Genet 2008;74:189-193.

11. Faiyaz-Ul-Haque M, Zaidi SH, Al-Sanna N, et al. A novel missense and recurrent mutation in SLC2A10 gene of patients affected with arterial tortuosity syndrome. Atherosclerosis 2009;203:466-471.

12. Davies RR, Kaple RK, Mandapati D, et al. Natural history of ascending aortic aneurysm in the setting of an unreplaced bicuspid aortic valve. Ann Thorac Surg 2007;83:1338-1344.

13. Garg V, Muth AN, Ransom JF, et al. Mutations in NOTCH1 cause aortic valve disease. Nature 2005;437:270-274.

14. Garg V. Molecular genetics of aortic valve disease. Curr Opin Cardiol 2006;21:180-184

15. McKellar SH, Tester DJ, Yagubyan M, Majumdar R, Ackerman MJ, Sundt TM III. Novel NOTCH1 mutations in patients with bicuspid aortic valve disease and thoracic aortic aneurysms. J Thorac Cardiovasc Surg 2007; 134:290-296.

16. Tadros TM, Klein MD, Shapiro OM. Ascending aortic dilatation associated with bicuspid aortic valve: pathophysiology, molecular biology, and clinical implications. Circulation 2009;119:880-890.

17. Janssens K, Vanhoenacker F, Bonduelle M, et al. Camurati-Engelmann disease: review of the clinical, radiological, and molecular data of 24 families and implications for diagnosis and treatment. J Med Genet 2006; 43:1-11.

18. Toydemir RM, Brassington AE, Bayrak-Toydemir P, et al. A novel mutation in FGFR3 causes camptodactyly, tall stature, and hearing loss (CATSHL) syndrome. Am J Hum Genet 2006;79:935-941.

19. Wang M, Clericuzio CL, Godfrey M. Familial occurrence of typical and severe lethal congenital contractural arachnodactyly caused by missplicing of exon 34 of fibrillin-2. Am J Hum Genet 1996;59:1027-1034.

20. Callewaert BL, Loeys BL, Ficcadenti A, et al. Comprehensive clinical and molecular assessment of 32 probands with congenital contractural arachnodactyly: report of 14 novel mutations and review of the literature. Hum Mutat 2009;30:334-341.

21. Frédéric MY, Monino C, Marschall C, et al. The FBN2 gene: new mutations, locus-specific database (Universal Mutation Database FBN2), and genotype-phenotype correlations. Hum Mutat 2009;30:181-190.

22. Nishimura A, Sakai H, Ikegawa S, et al. FBN2, FBN1, TGFBR1, and TGFBR2 analyses in congenital contractural arachnodactyly. Am J Med Genet A 2007;143:694-698.

23. Graul-Neumann LM, Hausser I, Essayie M, Rauch A, Kraus C. Highly variable cutis laxa resulting from a dominant splicing mutation of the elastin gene. Am J Med Genet A 2008;146A:977-983. 
24. Rodriguez-Revenga L, Iranzo P, Badenas C, Puig S, Carrió A, Milà M. A novel elastin gene mutation resulting in an autosomal dominant form of cutis laxa. Arch Dermatol 2004;140:1135-1139.

25. Szabo Z, Crepeau MW, Mitchell AL, et al. Aortic aneurysmal disease and cutis laxa caused by defects in the elastin gene. J Med Genet 2006;43:255258

26. Hucthagowder V, Sausgruber N, Kim KH, Angle B, Marmorstein LY, Urban Z. Fibulin-4: a novel gene for an autosomal recessive cutis laxa syndrome. Am J Hum Genet 2006;78:1075-1080.

27. Morava E, Guillard M, Lefeber DJ, Wevers RA. Autosomal recessive cutis laxa syndrome revisited. Eur J Hum Genet 2009;17:1099-1100.

28. Morava E, Lefeber D, Urban Z, et al. Defining the phenotype in an autosomal recessive cutis laxa syndrome with a combined congenital defect of glycosylation. Eur J Hum Genet 2008;16:28-35.

29. Guernsey DL, Jiang H, Evans SC, et al. Mutation in pyrroline-5-carboxylate reductase 1 gene in families with cutis laxa type 2. Am J Hum Genet 2009;85:120-129.

30. Reversade B, Escande-Beillard N, Dimopoulou A, et al. Mutations in PYCR1 cause cutis laxa with progeroid features. Nat Genet 2009;41:10161021

31. Kornak U, Reynders E, Dimopoulou A, et al. Impaired glycosylation and cutis laxa caused by mutations in the vesicular $\mathrm{H}+$-ATPase subunit ATP6V0A2. Nat Genet 2008;40:32-34.

32. Ali M, McKibbin M, Booth A, et al. Null mutations in LTBP2 cause primary congenital glaucoma. Am J Hum Genet 2009;84:664-671.

33. Ahram D, Sato TS, Kohilan A, et al. A homozygous mutation in ADAMTSL4 causes autosomal-recessive isolated ectopia lentis. Am J Hum Genet 2009;84:274-278.

34. Attias D, Stheneur C, Roy C, et al. Comparison of clinical presentations and outcomes between patients with TGFBR2 and FBN1 mutations in Marfan syndrome and related disorders. Circulation 2009:120:2541-2549.

35. Byers PH, Duvic M, Atkinson M, et al. Ehlers-Danlos syndrome type VIIA and VIIB result from splice-junction mutations or genomic deletions that involve exon 6 in the COL1A1 and COL1A2 genes of type I collagen. Am J Med Genet 1997;72:94-105.

36. Schwarze U, Hata R, McKusick VA, et al. Rare autosomal recessive cardiac valvular form of Ehlers-Danlos syndrome results from mutations in the COL1A2 gene that activate the nonsense-mediated RNA decay pathway. Am J Hum Genet 2004;74:917-930.

37. Malfait F, Symoens S, Coucke P, Nunes L, De Almeida S, De Paepe A. Total absence of the alpha2(1) chain of collagen type I causes a rare form of Ehlers-Danlos syndrome with hypermobility and propensity to cardiac valvular problems. J Med Genet 2006;43:E36.

38. Malfait F, De Paepe A. Molecular genetics in classics Ehlers-Danlos Syndrome. Am J Med Genet C Semin Med Genet 2005;139C:17-23.

39. Beighton P, De Paepe A, Steinmann B, Tsipouras P, Wenstrup RJ, et al. Ehlers-Danlos syndromes: revised nosology, Villefranche, 1997. EhlersDanlos National Foundation (USA) and Ehlers-Danlos Support Group (UK). Am J Med Genet 1998;77:31-37.

40. Malfait F, De Coster P, Hausser I, et al. The natural history, including orofacial features of three patients with Ehlers-Danlos syndrome, dermatosparaxis type (EDS type VIIC). Am J Med Genet A 2004;131: $18-28$

41. Colige A, Nuytinck L, Hausser I, et al. Novel types of mutation responsible for the dermatosparactic type of Ehlers-Danlos syndrome (Type VIIC) and common polymorphisms in the ADAMTS2 gene. J Invest Dermatol 2004; 123:656-663.

42. Voermans NC, van Engelen BG. Differential diagnosis of muscular hypotonia in infants: the kyphoscoliotic type of Ehlers-Danlos syndrome (EDS VI). Neuromuscul Disord 2008;18:906-907.

43. Yiçs U, Dirik E, Chambaz C, Steinmann B, Giunta C. Differential diagnosis of muscular hypotonia in infants: the kyphoscoliotic type of EhlersDanlos syndrome (EDS VI). Neuromuscul Disord 2008;18:210-214.

44. Salavoura K, Valari M, Kolialexi A, Mavrou A, Kitsiou S. A case of Ehlers Danlos syndrome type VI. Genet Couns 2006;17:291-294.

45. Coman DJ. The congenital disorders of glycosylation are clinical chameleons. Eur J Hum Genet 2008;16:2-4

46. Faiyaz-Ul-Haque M, Zaidi SH, Al-Ali M, et al. A novel missense mutation in the galactosyltransferase-I (B4GALT7) gene in a family exhibiting facioskeletal anomalies and Ehlers-Danlos syndrome resembling the progeroid type. Am J Med Genet A 2004;128A:39-45.

47. Giunta C, Elçioglu NH, Albrecht B, et al. Spondylocheiro dysplastic form of the Ehlers-Danlos Syndrome - an autosomal-recessive entity caused by mutations in the zinc transporter gene SLC39A13. Am J Hum Genet 2008:82:1290-1305.

48. Germain DP. Clinical and genetic features of vascular Ehlers-Danlos syndrome. Ann Vasc Surg 2002;16:391-397.

49. Snead MP, Yates JR, Williams R, Payne SJ, Pope FM, Scott JD. Stickler syndrome type 2 and linkage to the COL11A1 gene. Ann N Y Acad Sci 1996;785:331-332.

50. Plancke A, Holder-Espinasse M, Rigau V, Manouvrier S, Claustres M, Van
Kien PK. Homozygosity for a null allele of $C O L 3 A 1$ results in recessive Ehlers-Danlos syndrome. Eur J Hum Genet 2009;17:1411-1416.

51. Loeys BL, Schwarze U, Holm T, et al. Aneurysm syndromes caused by mutations in the TGF-beta receptor. $N$ Engl J Med 2006;355:788-798.

52. Voermans NC, van Alfen N, Pillen S, et al. Neuromuscular involvement in various types of Ehlers-Danlos syndrome. Ann Neurol 2009;65:687-697.

53. Bristow J, Carey W, Egging D, Schalkwijk J. Tenascin-X, collagen, elastin, and the Ehlers-Danlos syndrome. Am J Med Genet C Semin Med Genet 2005;139C:24-30.

54. Schalkwijk J, Zweers MC, Steijlen PM, et al. A recessive form of the Ehlers-Danlos syndrome caused by tenascin-X deficiency. $N$ Engl J Med 2001;345:1167-1175.

55. Lindor NM, Bristow J. Tenascin-X deficiency in autosomal recessive Ehlers-Danlos syndrome. Am J Med Genet A 2005;135:75-80.

56. Vaughan $\mathrm{C}$, Casey M, He J, et al. Identification of a chromosome 11q23.2q24 locus for familial aortic aneurysm disease, a genetically heterogeneous disorder. Circulation 2001;103:2469-2475

57. Guo DC, Pannu H, Tran-Fadulu V, et al. Mutations in smooth muscle alpha-actin (ACTA2) lead to thoracic aortic aneurysms and dissections. Nat Genet 2007;39:1488-1493

58. Tran-Fadulu VT, Pannu H, Kim DH, et al. Analysis of multigenerational families with thoracic aortic aneurysms and dissections due to TGFBR1 or TGFBR2 mutations. J Med Genet 2009;46:607-613.

59. Milewicz DM, Guo DC, Tran-Fadulu V, et al. Genetic basis of thoracic aortic aneurysms and dissections: focus on smooth muscle cell contractile dysfunction. Annu Rev Genomics Hum Genet 2008;9:283-302.

60. Davids JR, Hagerman RJ, Eilert RE. Orthopaedic aspects of fragile-X syndrome. J Bone Joint Surg Am 1990;72:889-896.

61. Hjalgrim H, Fisher Hansen B, Brondum-Nielson K, et al. Aspects of skeletal development in fragile $\mathrm{X}$ syndrome fetuses. Am J Med Genet 2000;95:123-9.

62. Garber KB, Visootsak J, Warren ST. Fragile X Syndrome. Eur J Hum Genet 2008;16:666-672.

63. Yap S. Classical homocystinuria: vascular risk and its prevention. $J$ Inherit Metab Dis 2003;26:259-265.

64. Salo AM, Cox H, Farndon P, et al. A connective tissue disorder caused by mutations of the lysyl hydroxylase 3 gene. Am J Hum Genet 2008;83:495503

65. Akutsu K, Morisaki H, Takeshita S, et al. Phenotypic heterogeneity of Marfan-like connective tissue disorders associated with mutations in the transforming growth factor-receptor genes. Circ $J$ 2007;71:1305-1309.

66. Lerma-Carrillo I, Molina JD, Cuevas-Duran T, et al. Psychopathology in the Lujan-Fryns syndrome: report of two patients and review. $\mathrm{Am} \mathrm{J} \mathrm{Med}$ Genet A 2006;140:2807-2811.

67. Van Buggenhout G, Fryns JP. Lujan-Fryns syndrome (mental retardation, X-linked, marfanoid habitus). Orphanet $J$ Rare Dis 2006;1:26.

68. Stathopulu E, Ogilvie CM, Flinter FA. Terminal deletion of chromosome $5 \mathrm{p}$ in a patient with phenotypical features of Lujan-Fryns syndrome. Am J Med Genet A 2003;119A:363-366.

69. Schwartz CE, Tarpey PS, Lubs HA, et al. The original Lujan syndrome family has a novel missense mutation (p.N1007S) in the MED12 gene. $J$ Med Genet 2007;44:472-477.

70. Blazek E, Mittler G, Meisterernst M. The mediator of RNA polymerase II. Chromosoma 2005;113:399-408.

71. Tarpey PS, Raymond FL, Nguyen LS, et al. Mutations in UPF3B, a member of the nonsense-mediated mRNA decay complex, cause syndromic and nonsyndromic mental retardation. Nat Genet 2007;39:11271133 .

72. Basel-Vanagaite L, Sarig O, Hershkovitz D, et al. RIN2 deficiency results in macrocephaly, alopecia, cutis laxa, and scoliosis: MACS syndrome. Am J Hum Genet 2009;85:254-263.

73. De Paepe A, Devereux R, Dietz HC, Hennekam RC, Pyeritz RE. Revised diagnostic criteria for the Marfan syndrome. Am J Med Genet 1996;62: 417-426.

74. Comeglio P, Johnson $P$, Arno $G$, et al. The importance of mutation detection in Marfan syndrome and Marfan-related disorders: report of 193 FBN1 mutations. Hum Mutat 2007;28:928.

75. Putnam EA, Cho M, Zinn AB, Towbin JA, Byers PH, Milewicz DM. Delineation of the Marfan phenotype associated with mutations in exons 23-32 of the FBN1 gene. Am J Med Genet 1996;62:233-242.

76. Pepe G, Giusti B, Evangelisti L, et al. Fibrillin-1 (FBN1) gene frameshift mutations in Marfan patients: genotype-phenotype correlation. Clin Genet 2001;59:444-450.

77. De Backer J, Loeys B, Leroy B, Coucke P, Dietz H, De Paepe A. Utility of molecular analyses in the exploration of extreme intrafamilial variability in the Marfan syndrome. Clin Genet 2007;72:188-198.

78. Glesby MJ, Pyeritz RE. Association of mitral valve prolapse and systemic abnormalities of connective tissue: a phenotypic continuum. JAMA 1989; 262:523-528

79. Freed LA, Levy D, Levine RA, et al. Prevalence and clinical outcome of mitral-valve prolapse. $N$ Engl J Med 1999;341:1-7. 
80. Grau JB, Pirelli L, Yu PJ, Galloway AC, Ostrer H. The genetics of mitral valve prolapse. Clin Genet 2007;72:288-295.

81. Khau Van Kien P, Wolf JE, Mathieu F, et al. Familial thoracic aortic aneurysm/dissection with patent ductus arteriosus: genetic arguments for a particular pathophysiological entity. Eur J Hum Genet 2004;12:173-180.

82. KhauVan Kien P, Mathieu F, Zhu L, et al. Mapping of familial thoracic aortic aneurysm/ dissection with patent ductus arteriosis to $16 \mathrm{p} 12.2$ p13.13. Circulation 2005;112:200-206.

83. Zhu L, Vranckx R, Khau Van Kien P, et al. Mutations in myosin heavy chain 11 cause a syndrome associating thoracic aortic aneurysm/aortic dissection and patent ductus arteriosus. Nat Genet 2006;38:343-349.

84. Zhu L, Bonnet D, Boussion M, Vedie B, Sidi D, Jeunemaitre X. Investigation of the MYH11 gene in sporadic patients with an isolated persistently patent arterial duct. Cardiol Young 2007; 17:666-672.

85. van Steensel MA, van Geel M, Parren LJ, Schrander-Stumpel CT, MarcusSoekarman D. Shprintzen-Goldberg syndrome associated with a novel missense mutation in TGFBR2. Exp Dermatol 2008;17:362-365.

86. Sood S, Eldadah ZA, Krause WL, McIntosh I, Dietz HC. Mutations in fibrillin-1 and the Marfanoid-craniosynostosis (Shprintzen-Goldberg) syndrome. Nat Genet 1996;12:209-211.

87. Kosaki K, Takahashi D, Udaka T, et al. Molecular pathology of ShprintzenGoldberg syndrome. Am J Med Genet A 2006;140:104-108.

88. Robinson PN, Neumann LM, Demuth S, et al. Shprintzen-Goldberg syndrome: fourteen new patients and a clinical analysis. Am J Med Genet A 2005; 135:251-262.

89. Becerra-Solano LE, Butler J, Castanedo-Cisneros G, et al. A missense mutation, p.V132G, in the X-linked spermine synthase gene (SMS) causes Snyder-Robinson syndrome. Am J Med Genet A 2009;149A:328-335.

90. Cason AL, Ikeguchi Y, Skinner C, et al. X-linked spermine synthase gene (SMS) defect: the first polyamine deficiency syndrome. Eur J Hum Genet 2003;11:937-944

91. de Alencastro G, McCloskey DE, Kliemann SE, et al. New SMS missense mutation leads to a striking reduction in spermine synthase protein function and a severe form of Snyder-Robinson X-linked recessive mental retardation syndrome. J Med Genet 2008;45:539-543.

92. Liberfarb RM, Levy HP, Rose PS, et al. The Stickler syndrome: genotype/ phenotype correlation in 10 families with Stickler syndrome resulting from seven mutations in the type II collagen gene locus COL2A1. Genet Med 2003;5:21-27.

93. Martin S, Richards AJ, Yates JR, Scott J, Pope M, Snead MP. Stickler syndrome: further mutations in COL11A1 and evidence for additional locus heterogeneity. Eur J Hum Genet 1999;7:807-814.

94. Donoso LA, Edwards AO, Frost AT, et al. Clinical variability of Stickler syndrome: role of exon 2 of the collagen COL2A1 gene. Surv Opthalmol 2003;48:191-203.

95. Sirko-Osadsa DA, Murray MA, Scott JA, Lavery MA, Warman ML, Robin NH. Stickler syndrome without eye involvement is caused by mutations in COL11A2, the gene encoding the alpha2(XI) chain of type XI collagen. J Pediatr 1998;132:368-371.

96. Dagoneau N, Benoist-Lasselin C, Huber C, et al. ADAMTS10 mutations in autosomal recessive Weill-Marchesani syndrome. Am J Hum Genet 2004; 75:801-806.

97. Faivre L, Dollfus H, Lyonnet S, et al. Clinical homogeneity and genetic heterogeneity in Weill-Marchesani syndrome. Am J Med Genet A 2003; 123A:204-207.

98. Kojuri J, Razeghinejad MR, Aslani A. Cardiac findings in WeillMarchesani syndrome. Am J Med Genet A 2007;143A:2062-2064.

99. Armstrong L, Graham GE, Schimke RN, et al. The Hunter-MacDonald syndrome with expanded phenotype including risk of meningioma: an update and review. Am J Med Genet A 2008;146A:83-92.

100. Gelb BD. Marfan's syndrome and related disorders-more tightly connected than we thought. $N$ Engl J Med 2006;355:841-844.

101. Matt P, Habashi J, Carrel T, Cameron DE, Van Eyk JE, Dietz HC. Recent advances in understanding Marfan syndrome: should we now treat surgical patients with losartan? J Thorac Cardiovasc Surg 2008;135:389-394. 